\title{
Heat shock protein 90 as a long-term buffer of mutational burden
}

\author{
Valeria Timonina* \\ Center for Mitochondrial Functional \\ Genomics, Immanuel Kant Baltic \\ Federal University \\ Kaliningrad, Russia \\ valeratimonina@gmail.com \\ Evgenii Tretiakov \\ Department of Molecular \\ Neurosciences, Medical University of \\ Vienna, Vienna, Austria \\ evgenii.o.tretiakov@gmail.com
}

\author{
Anastasia Sokol* \\ Center for Mitochondrial Functional \\ Genomics, Immanuel Kant Baltic \\ Federal University \\ Kaliningrad, Russia \\ anastasia3sokol@yandex.ru \\ Konstantin Gunbin \\ Institute of Cytology and Genetics SB \\ RAS, Novosibirsk, Russia \\ genkvg@gmail.com
}

\author{
Dmitry Knorre \\ Belozersky Institute Of \\ Physico-Chemical Biology \\ Moscow State University \\ Moscow, Russia \\ knorre@belozersky.msu.ru \\ Konstantin Popadin \\ School of Life Sciences \\ Ecole Polytechnique Federale de \\ Lausanne, Lausanne, Switzerland \\ Center for Mitochondrial Functional \\ Genomics, Immanuel Kant Baltic \\ Federal University \\ Kaliningrad, Russia \\ konstantinpopadin@gmail.com
}

\begin{abstract}
*- this authors contributed equally to this work
Abstract - Heat shock proteins play an important role in protein homeostasis of cells maintaining the correct folding of disturbed proteins. Several lines of evidence suggest the role of hsp90 in buffering mutational burden in model organisms. Here we propose a hypothesis that hsp90 may act as a longterm buffer of mutational load influencing species evolution. To prove the hypothesis we use interspecies comparison of expression and genomic data.
\end{abstract}

Keywords - hsp90, mutation buffering, evolutionary constraints

\section{Introduction}

Heat shock proteins (hsp) maintain optimal folding of their clients - proteins, affected by environmental (temperature) or genetic (a load of slightly deleterious mutations) perturbations. In several short-term experiments (typically from one to several generations) it has been shown that hsp90 acts as a buffer of the mutational burden influencing the manifestation of slightly-deleterious variants [1-4]. Here, we propose that hsp90 may play also a long-term evolutionary role compensating, for example, an increased mutational burden in species with low effective population size $(\mathrm{Ne})$. Thus hsp90 might be under stronger evolutionary constraints in low-sized versus high-sized populations.

\section{Results}

Taking into account that the number of fixed slightly deleterious nonsynonymous variants is higher in species with low effective population size $[5,6]$, we expect that the expression level of hsp90 in these species is increased to partially compensate the high burden of slightly-deleterious nonsynonymous variants (SDNVs). Indeed using comparative species expression level data [8] we found out that expression level of hsp90 is the lowest in Macaque, which is characterized by the highest effective population size among other six analyzed [8] primates (Ne of macaque

80'000, Yuan et al.2012; human: 13'100-16'200; gorilla: 28'400-56'900; chimp: 30'900-61'800; orangutan: $42^{\prime} 300-84$ '600, data from [9]).
Analyses of hsp90 expression pattern between different human tissues (the highest in the ovary, GTEX portal https://www.gtexportal.org/) and different stages of mammalian development (the highest expression is observed at early stages of development when the most essential genes are expressed) [9] is in line with supportive role of hsp90 during the development.

Next, we assumed that because of this potential compensatory role, hsp90 might be under stronger evolutionary constraints in low-sized versus high-sized populations. In order to compare the level of relaxation of hsp90 in different mammalian species, we performed a linear regression analysis with scaling of variables between species-specific $\mathrm{Kn} / \mathrm{Ks}$ of hsp90 (using only 1-to-1 orthologs of human Hsp90) and species-specific generation length (which approximates the effective population size) of 38 mammalian species. We obtained weak positive correlation ( slope $=0.2864$, $\mathrm{p}$-value $=0.05$, $\mathrm{R}^{\wedge} 2=0.082$ ) which is in line with our expectations (Fig 1.). In order to make the final conclusion about selection relaxation of hsp90 we compared the hsp90 specific slope with slopes of genes similar to hsp90. To derive a subset of genes comparable to hsp90 we performed Principal Component Analysis taking into account evolutionary characteristics of human genes such as the probability of being Loss of Function intolerant, haploinsufficiency [7], etc. as well as structural features like average exon length, gene length, number of transctripts, etc. 


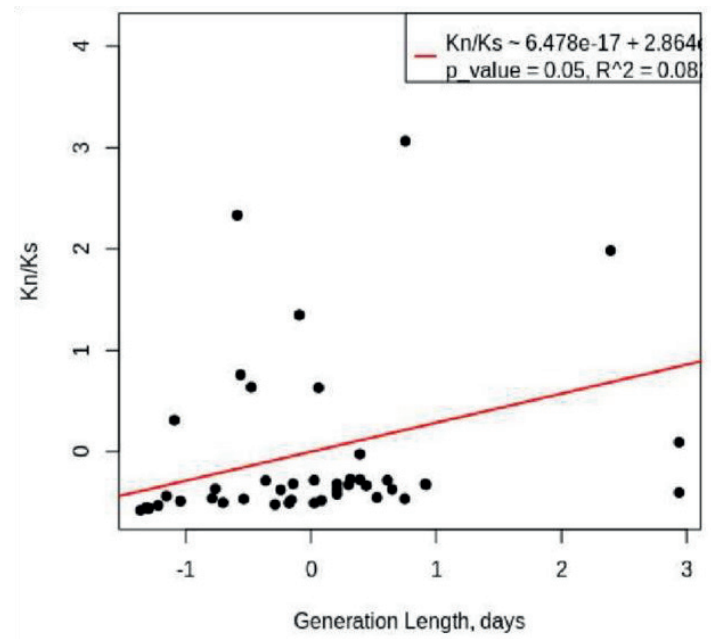

Fig. 1. Linear regression of $\mathrm{Kn} / \mathrm{Ks}$ vs Generation Length for hsp90

We expect that the hsp90-specific slope should be less steep compared to other genes. Across comparable genes, hsp90 has one of the lowest mean $\mathrm{Kn} / \mathrm{Ks}$ that emphasizes its high level of evolutionary constraints. However detailed analysis of slopes from linear regression models of $\mathrm{Kn} / \mathrm{Ks}$ vs Generation length did not show robust signal of the decreased hsp90-specific slope versus the slopes of other genes.

Finally, we analyzed the distribution of cis-eQTLs (GTEX portal - https://www.gtexportal.org/) in hsp90 compared to a subset of similar genes (see the previous section). We observed that hsp90 has a significantly lower assessed allele frequency of cis-eQTLs than in other genes (Mann-Whitney test, $\mathrm{W}=88256$, $\mathrm{p}$-value $=0.025$ ). This fact suggests that hsp90 due to its role in homeostasis maintenance should keep a constant expression level and so it has a deficit of regulatory expression variants compared to other genes.

Altogether we observed several lines of evidence, confirming the compensatory role of hsp90 but more analyses are needed to confirm it's long-term bufferisation effect.

\section{Acknowledgments}

This work has been supported by the 5 Top 100 Russian Academic Excellence Project at the Immanuel Kant Baltic Federal University, by the Russian Foundation for Basic Research grant 18-29-13055.

\section{References}

[1] Fares, M. A., Ruiz-González, M. X., Moya, A., Elena, S. F., \& Barrio, E. (2002). GroEL buffers against deleterious mutations. Nature, 417(6887), 398-398.

[2] Sangster, T. A., Salathia, N., Lee, H. N., Watanabe, E., Schellenberg, K., Morneau, K., ... \& Lindquist, S. (2008). HSP90-buffered genetic variation is common in Arabidopsis thaliana. Proceedings of the National Academy of Sciences, 105 (8), 2969-2974.

[3] Rutherford, Suzanne L., and Susan Lindquist. "Hsp90 as a capacitor for morphological evolution." Nature 396.6709 (1998): 336. Maisnier-Patin, S., Roth, J., Fredriksson, A. et al. Genomic buffering mitigates the effects of deleterious mutations in bacteria. Nat Genet 37, 1376-1379 (2005).

[4] Popadin, K., Polishchuk, L. V., Mamirova, L., Knorre, D., \& Gunbin, K. (2007). Accumulation of slightly deleterious mutations in mitochondrial protein-coding genes of large versus small mammals. Proceedings of the National Academy of Sciences, 104(33), 13390-13395.

[5] Popadin, K. Y., Gutierrez-Arcelus, M., Lappalainen, T., Buil, A., Steinberg, J., Nikolaev, S. I., Antonarakis, S. E. (2014). Gene age predicts the strength of purifying selection acting on gene expression variation in humans. American Journal of Human Genetics95(6), 660-674.

[6] Bartha, I., di Iulio, J., Venter, J. et al. Human gene essentiality. Nat Rev Genet 19, 51-62 (2018). https://doi.org/10.1038/nrg.2017.75

[7] Brawand, D., Soumillon, M., Necsulea, A., Julien, P., Csárdi, G., Harrigan, P., ... \& Albert, F. W. (2011). The evolution of gene expression levels in mammalian organs. Nature, 478(7369), 343-348.

[8] Prado-Martinez, J., Sudmant, P. H., Kidd, J. M., Li, H., Kelley, J. L., Lorente-Galdos, B., ... \& Cagan, A. (2013). Great ape genetic diversity and population history. Nature, 499(7459), 471-475.

[9] Cardoso-Moreira, M., Halbert, J., Valloton, D. et al. Gene expression across mammalian organ development. Nature 571, 505-509 (2019). 